\title{
Energy Conditions in Palatini Approach to Modified $f(R)$ Gravity
}

\author{
Hamidreza Saiedi \\ Department of Physics, Florida Atlantic University, Boca Raton, FL, USA \\ Email: hsaiedi2014@fau.edu,hrssaiedi@gmail.com
}

How to cite this paper: Saiedi, H. (2020) Energy Conditions in Palatini Approach to Modified $f(R)$ Gravity. Journal of High Energy Physics, Gravitation and Cosmology, 6, 671-679.

https://doi.org/10.4236/jhepgc.2020.64046

Received: July 13, 2020

Accepted: September 26, 2020

Published: September 29, 2020

Copyright $\odot 2020$ by author(s) and Scientific Research Publishing Inc. This work is licensed under the Creative Commons Attribution International License (CC BY 4.0).

http://creativecommons.org/licenses/by/4.0/

\section{(c) (i) Open Access}

\begin{abstract}
In this paper, we review modified $f(R)$ theories of gravity in Palatini formalism. In this framework, we use the Raychaudhuri's equation along with the requirement that the gravity is attractive, which holds for any geometrical theory of gravity to discuss the energy conditions. Then, to derive these conditions, we obtain an expression for effective pressure and energy density by considering FLRW metric. To simply express the energy conditions, we write the Ricci scalar and its derivatives in terms of the deceleration $(q)$, jerk $(j)$ and snap $(s)$ parameters. Energy conditions derived in Palatini version of $f(R)$ Gravity differ from those derived in GR. We will see that the WEC (weak energy condition) derived in Palatini formalism has exactly the same expression in its metric approach.
\end{abstract}

\section{Keywords}

Modified Gravity, Palatini Formalism, Energy Conditions

\section{Introduction}

According to astronomical observations, the gravity force at large scales may not behave in standard GR derived from the Hilbert-Einstein action,

$$
A=\frac{1}{16 \pi G} \int \sqrt{-g} R \mathrm{~d}^{4} x+\int \sqrt{-g} L_{m} \mathrm{~d}^{4} x,
$$

where $R$ is the Ricci scalar, $G$ is Newton's gravitational constant, and $L_{m}$ is the matter lagrangian density, respectively [1] [2] [3] [4]. So, a generalized Hilbert-Einstein action may be required to fully understand the gravitational interaction. One of the possible ways to generalize GR is related to the modification of the geometric section of Hilbert-Einstein action. Examples of such modified gravity models are introduced in [5] [6], by assuming that the Ricci scalar $R$ in 
the lagrangian is replaced by an arbitrary function $f(R)$ of the Ricci scalar. For discussions of modified $f(R)$ gravity theories see [7]-[19]. The modified $f(R)$ theories of gravity can easily explain the recent cosmological observations, and give a solution to the dark matter problem [20] [21]. The metric and Palatini formalisms are two different ways that GR can be derived and lead to the same field equations [22]. However, in modified $f(R)$ gravity, the equations of motion in Palatini approach and metric formalism are generically different [22]. The field equations in metric approach are higher-order while in Palatini approach they are second-order. Both these formalisms in $f(R)$ theories allow the formulation of simple extensions of Einstein's GR. For further discussions of $f(R)$ theories of gravity involving geometry and matter coupling see [23] [24].

In the cosmological context, different $f(R)$ models give rise to the problem of how to constrain from theoretical and observational aspects of these possible $f(R)$ models. Recently, by testing the cosmological viability of some specific cases of $f(R)$ this possibility has been discussed [25]-[31]. By imposing the energy conditions, we may have further constrains to $f(R)$ theories of gravity [32] [33]. In different contexts, these conditions (so-called energy conditions) have been used to obtain global solutions for a variety of situations. As an example, the weak energy condition (WEC) and strong energy condition (SEC) were used in the Hawking-Penrose singularity theorems. Also, the null energy condition (NEC) is required to prove the second law of black hole thermodynamics. However, the energy conditions were basically formulated in GR [34], one can drive these conditions in $f(R)$ theories of gravity by introducing new effective pressure and energy density defined in Jordan frame. In the present paper, the energy conditions for $f(R)$ theories in Palatini formalism are derived by using the Raychaudhuri's Equation (along with the attraction of gravity) which is the ultimate origin of the energy conditions.

\section{Palatini Formalism for $f(R)$ Theories of Gravity}

To explain the cosmic speed-up, the model $f(R)=R-\mu^{4} / R$ in metric formalism has some problems. By observation that these problems could be avoided by considering its Palatini formalism, this approach to $f(R)$ theories of gravity has been boosted. Also, the energy conditions in GR and metric formalism of $f(R)$ theories have been discussed in different contexts. So, finding the energy conditions in Palatini version of $f(R)$ would be interesting that we will discuss them in the present paper. Therefore, first we review the field equations in Palatini formalism, and then obtain the energy conditions. The action that defines $f(R)$ theories has the generic form

$$
A=\frac{1}{2 k^{2}} \int \sqrt{-g} f(R) \mathrm{d}^{4} x+A_{m}\left[g_{\mu v}, \psi_{m}\right]
$$

where $A_{m}\left[g_{\mu \nu}, \psi_{m}\right]$ represents the matter action, which depends on the metric $g_{\mu v}$ and the matter field $\psi_{m}$. In the case of Palatini formalism, the connection 
$\Gamma_{\mu \nu}^{\lambda}$ and the metric $g_{\mu \nu}$ are regarded as dynamical variables to be independently varied. Varying the action respect to the metric does yield dynamical equations

$$
f^{\prime}(R) R_{\mu \nu}(\Gamma)-\frac{1}{2} f(R) g_{\mu v}=k^{2} T_{\mu \nu}
$$

where $f^{\prime}(R)=\frac{\mathrm{d} f}{\mathrm{~d} R}$ and $T_{\mu \nu}$ is the usual energy-momentum tensor. $R_{\mu \nu}(\Gamma)$ is the Ricci tensor corresponding to the connection $\Gamma_{\mu \nu}^{\lambda}$, which is in general different from the Ricci tensor corresponding to the metric connection $R_{\mu v}(g)$. Taking the trace of the Equation (2), we obtain

$$
f^{\prime}(R) R-2 f(R)=k^{2} T,
$$

where $R=R(T)=g^{\mu v} R_{\mu v}(\Gamma)$ is directly related to $T$ and is different from the Ricci scalar $R(g)=g^{\mu v} R_{\mu \nu}(g)$ in the metric case. Varying the action (1) with respect to the connection yields

$$
\nabla_{\alpha}\left(\sqrt{-g} f^{\prime}(R) g^{\mu \nu}\right)=0,
$$

Taking into account that under conformal transformations how the Ricci tensor transforms, it has been shown that [22] [35]

$$
\begin{aligned}
& R_{\mu \nu}(g)-\frac{1}{2} g_{\mu \nu} R(g) \\
& =\frac{k^{2}}{f^{\prime}} T_{\mu \nu}-\frac{R(T) f^{\prime}-f}{2 f^{\prime}} g_{\mu v}+\frac{1}{f^{\prime}}\left(\nabla_{\mu} \nabla_{v} f^{\prime}-g_{\mu \nu} \square f^{\prime}\right) \\
& -\frac{3}{2\left(f^{\prime}\right)^{2}}\left[\partial \mu f^{\prime} \partial v f^{\prime}-\frac{1}{2} g_{\mu \nu}\left(\partial f^{\prime}\right)^{2}\right]
\end{aligned}
$$

where $R_{\mu v}(g)$ and $R(g)$ are computed in terms of the Levi-Civita connection of the metric $g_{\mu \nu}$, i.e., they represent the usual Ricci tensor and scalar curvature. It follows that $R(T)=g^{\mu \nu} R_{\mu \nu}(\Gamma)$ and $R(g)=g^{\mu v} R_{\mu \nu}(g)$ are related by

$$
R=R(T)=R(g)+\frac{3}{2\left(f^{\prime}\right)^{2}} \partial_{\lambda} f^{\prime} \partial^{\lambda} f^{\prime}-\frac{3}{f^{\prime}} \square f^{\prime}
$$

For simplicity, we take $k^{2}=8 \pi G=1$. Now, we can realize that the right hand side of Equation (5) can be considered as an effective energy-momentum tensor $T_{\mu v}^{e}$. So

$$
\begin{aligned}
T_{\mu \nu}^{e}= & \frac{1}{f^{\prime}} T_{\mu \nu}-\frac{R(T) f^{\prime}-f}{2 f^{\prime}} g_{\mu \nu}+\frac{1}{f^{\prime}}\left(\nabla_{\mu} \nabla_{\nu} f^{\prime}-g_{\mu \nu} \square f^{\prime}\right) \\
& -\frac{3}{2\left(f^{\prime}\right)^{2}}\left[\partial \mu f^{\prime} \partial v f^{\prime}-\frac{1}{2} g_{\mu \nu}\left(\partial f^{\prime}\right)^{2}\right] .
\end{aligned}
$$

Taking the trace of the above equation, one can easily find

$$
T^{e}=g^{\mu v} T_{\mu \nu}^{e}=\frac{T}{f^{\prime}}-\frac{2}{f^{\prime}}\left(R(T) f^{\prime}-f\right)-\frac{3 \square f^{\prime}}{f^{\prime}}+\frac{3}{2\left(f^{\prime}\right)^{2}}\left(\partial f^{\prime}\right)^{2} .
$$


By substituting $T$ from (3) into Equation (8), after simplification, we reach

$$
T^{e}=\frac{3}{2\left(f^{\prime}\right)^{2}}\left(\partial f^{\prime}\right)^{2}-\frac{3 \square f^{\prime}}{f^{\prime}}-R(T) .
$$

Now, by comparing the above relation and Equation (6), one can easily realize that

$$
T^{e}=-R(g) .
$$

So, we can rewrite the Equation (5) as

$$
R_{\mu v}(g)=T_{\mu \nu}^{e}-\frac{T^{e}}{2} g_{\mu v},
$$

where $T_{\mu \nu}^{e}$ and $T^{e}$ are Equations (7) and (8), respectively.

\section{Energy Conditions in Palatini Version of $f(R)$}

To find the energy conditions, we shall use the Raychaudhuri's equation which holds for any geometrical theory of gravity. Therefore, we first briefly review these conditions in GR and then apply them to the $f(R)$ modified gravity in Palatini formalism. The Raychaudhuri's equation implies that for any hypersurface orthogonal congruences, the condition for attractive gravity (convergence of time like geodesics) reduces to $R_{\mu \nu}(g) u^{\mu} u^{v} \geq 0$, where $u^{\mu}$ is a tangent vector field to a congruence of time like geodesics. In GR, using the units such that $k^{2}=8 \pi G=c=1$, we have $R_{\mu \nu}(g)-\frac{1}{2} g_{\mu \nu} R(g)=T_{\mu \nu}$ or $R_{\mu v}(g)=T_{\mu v}-\frac{T}{2} g_{\mu v}$.

So the condition $R_{\mu \nu}(g) u^{\mu} u^{v} \geq 0$ implies that

$$
R_{\mu v}(g) u^{\mu} u^{v}=\left(T_{\mu \nu}-\frac{T}{2} g_{\mu v}\right) u^{\mu} u^{v} \geq 0 .
$$

For a perfect fluid with energy density $\rho$ and pressure $p$

$$
T_{\mu \nu}=(\rho+p) u_{\mu} u_{v}-p g_{\mu v} .
$$

by using the restriction (12), the SEC can be written as $(\rho+3 p) \geq 0$.

The condition for convergence of null geodesics along with Einsteins's equations leads to

$$
R_{\mu v}(g) k^{\mu} k^{v}=T_{\mu \nu} k^{\mu} k^{v} \geq 0 .
$$

which is the NEC. Here $k^{\mu}$ is a tangent vector field to a congruence of null geodesics. Therefore, the NEC for the energy-momentum tensor (13) can be written as $(\rho+p) \geq 0$.

Since, the Raychaudhuri's equation is valid for any geometrical gravity theory, so, the conditions $R_{\mu v}(g) u^{\mu} u^{v} \geq 0$ and $R_{\mu v}(g) k^{\mu} k^{v} \geq 0$ along with field equations in Palatini version of $f(R)$ gravity implies that

$$
R_{\mu v}(g) u^{\mu} u^{v}=\left(T_{\mu v}^{e}-\frac{T^{e}}{2} g_{\mu v}\right) u^{\mu} u^{v} \geq 0,
$$




$$
R_{\mu v}(g) k^{\mu} k^{v}=T_{\mu \nu}^{e} k^{\mu} k^{v} \geq 0 .
$$

where we have used $T_{\mu \nu}^{e}$ instead of $T_{\mu \nu}$. Now, by comparing the above equations with Equations (12) and (14), we can simply figure out that the SEC and NEC can be modified as $\left(\rho_{e}+3 p_{e}\right) \geq 0$ and $\left(\rho_{e}+p_{e}\right) \geq 0$, respectively.

By substituting (6) into (7), for the homogeneous and isotropic FriedmannLemaitre-Robertson-Walker (FLRW) metric with scale factor $a(t)$, and after some simplifications, we reach the following relations for effective energy density $\rho_{e}$ and effective pressure $p_{e}$.

$$
\begin{gathered}
\rho_{e}=T_{0}^{0 e}=\frac{1}{f^{\prime}}\left[\rho+\frac{1}{2}\left(f-R(g) f^{\prime}\right)-\frac{3}{2 f^{\prime}}\left(\partial_{0} f^{\prime}\right)^{2}+\frac{3}{2} \partial_{0} \partial_{0} f^{\prime}+\frac{3}{2} H \partial_{0} f^{\prime}\right], \\
p_{e}=-T_{1}^{1 e}=\frac{1}{f^{\prime}}\left[p-\frac{1}{2}\left(f-R(g) f^{\prime}\right)-\frac{1}{2} \partial_{0} \partial_{0} f^{\prime}-\frac{5}{2} H \partial_{0} f^{\prime}\right] .
\end{gathered}
$$

Here, $H=\dot{a} / a$ is the Hubble parameter. We can easily rewrite the above equations as (by denoting $R_{g}=R(g)$ )

$$
\begin{gathered}
\rho_{e}=\frac{1}{f^{\prime}}\left[\rho+\frac{1}{2}\left(f-R_{g} f^{\prime}\right)\right]+\frac{1}{f^{\prime}}\left[\frac{3}{2} \ddot{R}_{g} f^{\prime \prime}-\frac{3}{2 f^{\prime}} \dot{R}_{g}^{2} f^{\prime \prime 2}+\frac{3}{2} \dot{R}_{g}^{2} f^{\prime \prime \prime}+\frac{3}{2} H \dot{R}_{g} f^{\prime \prime}\right], \\
p_{e}=\frac{1}{f^{\prime}}\left[p-\frac{1}{2}\left(f-R_{g} f^{\prime}\right)-\frac{1}{2} \ddot{R}_{g} f^{\prime \prime}-\frac{1}{2} \dot{R}_{g}^{2} f^{\prime \prime \prime}-\frac{5}{2} H \dot{R}_{g} f^{\prime \prime}\right] .
\end{gathered}
$$

To simply express the energy conditions, we can write the Ricci scalar and its derivatives for a spatially flat FLRW metric in terms of the deceleration $(q)$, jerk $(j)$ and snap $(s)$ parameters [36] [37] [38]

$$
\begin{aligned}
& R_{g}=-6 H^{2}(1-q) \\
& \dot{R}_{g}=-6 H^{3}(j-q-2) \\
& \ddot{R}_{g}=-6 H^{4}\left(s+q^{2}+8 q+6\right)
\end{aligned}
$$

where

$$
q=-\frac{1}{a H^{2}} \cdot \frac{\mathrm{d}^{2} a}{\mathrm{~d} t^{2}}, \quad j=\frac{1}{a H^{3}} \cdot \frac{\mathrm{d}^{3} a}{\mathrm{~d} t^{3}}, \quad s=\frac{1}{a H^{4}} \cdot \frac{\mathrm{d}^{4} a}{\mathrm{~d} t^{4}}
$$

Now, we can classify the energy conditions as follow

NEC: $\left(\rho_{e}+p_{e}\right) \geq 0$

$$
\begin{aligned}
& \rho+p+\ddot{R}_{g} f^{\prime \prime}-\frac{3}{2 f^{\prime}} \dot{R}_{g}^{2} f^{\prime \prime 2}+\dot{R}_{g}^{2} f^{\prime \prime \prime}-H \dot{R}_{g} f^{\prime \prime} \geq 0 \Rightarrow \\
& \rho+p-6 H^{4}\left(s+q^{2}+8 q+6\right) f^{\prime \prime}-\frac{54}{f^{\prime}} H^{6}(j-q-2)^{2} f^{\prime \prime 2} \\
& +36 H^{6}(j-q-2)^{2} f^{\prime \prime \prime}+6 H^{4}(j-q-2) f^{\prime \prime} \geq 0
\end{aligned}
$$

SEC: $\left(\rho_{e}+3 p_{e}\right) \geq 0$

$$
\rho+3 p-f+R_{g} f^{\prime}-\frac{3}{2 f^{\prime}} \dot{R}_{g}^{2} f^{\prime \prime 2}-6 H \dot{R}_{g} f^{\prime \prime} \geq 0 \Rightarrow
$$




$$
\begin{aligned}
& \rho+3 p-f-6 H^{2}(1-q) f^{\prime}-\frac{54}{f^{\prime}} H^{6}(j-q-2)^{2} f^{\prime \prime 2} \\
& +36 H^{4}(j-q-2) f^{\prime \prime} \geq 0
\end{aligned}
$$

WEC (week energy condition): beside the inequality (23), $\rho_{e} \geq 0$

$$
\begin{aligned}
& \rho+\frac{1}{2}\left(f-R_{g} f^{\prime}\right)-3 H \dot{R}_{g} f^{\prime \prime} \geq 0 \Rightarrow \\
& \rho+\frac{1}{2} f+3 H^{2}(1-q) f^{\prime}+18 H^{4}(j-q-2) f^{\prime \prime} \geq 0
\end{aligned}
$$

DEC (dominant energy condition): beside the inequalities (23) and (25), $\left(\rho_{e}-p_{e}\right) \geq 0$

$$
\begin{aligned}
& \rho-p+f-R_{g} f^{\prime}+2 \dot{R}_{g}^{2} f^{\prime \prime \prime}-\frac{3}{2 f^{\prime}} \dot{R}_{g}^{2} f^{\prime \prime 2}+2 \ddot{R}_{g} f^{\prime \prime}+4 H \dot{R}_{g} f^{\prime \prime} \geq 0 \Rightarrow \\
& \rho-p+f+6 H^{2}(1-q) f^{\prime}+72 H^{6}(j-q-2)^{2} f^{\prime \prime \prime}-\frac{54}{f^{\prime}} H^{6}(j-q-2)^{2} f^{\prime \prime 2} \\
& -12 H^{4}\left(s+q^{2}+8 q+6\right) f^{\prime \prime}-24 H^{4}(j-q-2) f^{\prime \prime} \geq 0
\end{aligned}
$$

It is useful to discuss the energy conditions for some specific $f(R)$ models for the present values of deceleration, jerk, and snap parameters. As we can see from the inequalities (23), (24), (25), and (26), these inequalities depend on the value of the snap parameter except for WEC and SEC. Since a reliable value of this parameter has not been reported, therefore, only the WEC and SEC are discussable with the present observational datas of deceleration and jerk parameters $\left(q_{0}=-0.81, j_{0}=2.16\right)$. We shall note that the inequality (25) for WEC is exactly the same inequality found in [30]. The authors of [30] perfectly explain the WEC for $R+\mu R^{n}$ and $\mu R^{n}$ models of $f(R)$ gravity.

\section{Conclusion}

In the context of modified $f(R)$ gravity, we have reviewed the field equations in Palatini formalism. It is shown that the model $f(R)=R-\mu^{4} / R$ in metric approach has some problems to explain the cosmic speed-up. By considering the Palatini version of this model, these problems can be avoided. To discuss the energy conditions, we use the Raychaudhuri's equation along with the requirement that the gravity is attractive, which is the ultimate origin of the energy conditions and holds for any geometrical theory of gravity. We consider FLRW metric to derive the effective pressure and energy density, which are needed to find the energy conditions. It is seen that these conditions are different from those derived in the context of GR. We have shown that the WEC and SEC are independent of the snap parameter $(s)$. It is worth to mention here that the WEC derived in Palatini formalism of $f(R)$ gravity is exactly the same WEC found in its metric approach. We should note that in the context of general relativity all these energy conditions seem to be violated at some points.

\section{Conflicts of Interest}

The author declares no conflicts of interest regarding the publication of this paper. 


\section{References}

[1] Perlmutter, S., et al. (1999) Measurements of $\Omega$ and $\Lambda$ from 42 High-Redshift Supernovae. The Astrophysical Journal, 517, 565-586. https://doi.org/10.1086/307221

[2] Amanullah, R., et al. (2010) Spectra and Light Curves of Six Type Ia Supernovae at $0.511<\mathrm{z}<1.12$ and the Union2 Compilation. The Astrophysical Journal, 716, 712-738. https://doi.org/10.1088/0004-637X/716/1/712

[3] Overduin, J.M. and Wesson, P.S. (2004) Dark Matter and Background Light. Physics Reports, 402, 267-406. https://doi.org/10.1016/j.physrep.2004.07.006

[4] Baer, H., Choi, K.-Y., Kim, J.E. and Roszkowski, L. (2015) Dark Matter Production in the Early Universe: Beyond the Thermal WIMP Paradigm. Physics Reports, 555, 1-60. https://doi.org/10.1016/j.physrep.2014.10.002

[5] Buchdahl, H.A. (1970) Non-Linear Lagrangians and Cosmological Theory. Monthly Notices of the Royal Astronomical Society, 150, 1-8. https://doi.org/10.1093/mnras/150.1.1

[6] Barrow, J.D. and Ottewill, A.C. (1983) The Stability of General Relativistic Cosmological Theory. Journal of Physics A: Mathematical and General, 16, 2757. https://doi.org/10.1088/0305-4470/16/12/022

[7] Carroll, S.M., Duvvuri, V., Trodden, M. and Turner, M.S. (2004) Is Cosmic Speed-Up Due to New Gravitational Physics? Physical Review D, 70, Article ID: 043528. https://doi.org/10.1103/PhysRevD.70.043528

[8] Nojiri, S. and Odintsov, S.D. (2007) Introduction to Modified Gravity and Gravitational Alternative for Dark Energy. International Journal of Geometric Methods in Modern Physics, 4, 115-145. https://doi.org/10.1142/S0219887807001928

[9] Sotiriou, T.P. and Faraoni, V. (2010) $f(\mathrm{R})$ Theories of Gravity. Reviews of Modern Physic, 82, 451. https://doi.org/10.1103/RevModPhys.82.451

[10] Capozziello, S. and De Laurentis, M. (2011) Extended Theories of Gravity. Physics Reports, 509, 167-321. https://doi.org/10.1016/j.physrep.2011.09.003

[11] Nojiri, S., Odintsov, S.D. and Oikonomou, V.K. (2017) Modified Gravity Theories on a Nutshell: Inflation, Bounce and Late-Time Evolution. Physics Reports, 692, 1-104. https://doi.org/10.1016/j.physrep.2017.06.001

[12] Bahamonde, S., Jamil, M., Pavlovic, P. and Sossich, M. (2016) Cosmological Wormholes in $f(\mathrm{R})$ Theories of Gravity. Physical Review D, 94, Article ID: 044041. https://doi.org/10.1103/PhysRevD.94.044041

[13] Saiedi, H. and Nasr Esfahani, B. (2011) Time-Dependent Wormhole Solutions of $f(\mathrm{R})$ Theory of Gravity and Energy Conditions. Modern Physics Letters A, 26, 1211-1219. https://doi.org/10.1142/S0217732311035547

[14] Dutta, J., Mitra, S. and Chetry, B. (2016) Modified Bekenstein-Hawking System in $f(\mathrm{R})$ Gravity. International Journal of Theoretical Physics, 55, 4272-4285. https://doi.org/10.1007/s10773-016-3052-5

[15] Habib Mazharimousavi, S. and Halilsoy, M. (2016) Necessary Conditions for Having Wormholes in $f(\mathrm{R})$ Gravity. Modern Physics Letters A, 31, 16502035. https://doi.org/10.1142/S0217732316502035

[16] Saiedi, H. (2012) Thermodynamics of Evolving Lorentzian Wormholes at Apparent Horizon in $f(\mathrm{R})$ Theory of Gravity. Modern Physics Letters A, 27, 1250220. https://doi.org/10.1142/S0217732312502203

[17] Pasqua, A., Chattopadhyay, S. and Myrzakulov, R. (2013) A Dark Energy with Higher order Derivatives of $\mathrm{H}$ in the Modified Gravity $f(\mathrm{R}, \mathrm{T})$. ISRN High Energy Physics, 2014, Article ID: 535010. https://doi.org/10.1155/2014/535010 
[18] Saiedi, H. (2017) Modified $f(\mathrm{R})$ Gravity and Thermodynamics of Time-Dependent Wormholes at Event Horizon. Journal of High Energy Physics, Gravitation and Cosmology, 3, 708-714. https://doi.org/10.4236/jhepgc.2017.34053

[19] Xu, T., Cao, S., Qi, J., Biesiada, M., Zheng, X. and Zhu, Z.-H. (2018) A New Test of $f(\mathrm{R})$ Gravity with the Cosmological Standard Rulers in Radio Quasars. Journal of Cosmology and Astroparticle Physics, 2018, 1-19. https://doi.org/10.1088/1475-7516/2018/06/042

[20] Boehmer, C.G., Harko, T. and Lobo, F.S.N. (2008) Dark Matter as a Geometric Effect in $f(\mathrm{R})$ Gravity. Astroparticle Physics, 29, 386-392.

https://doi.org/10.1016/j.astropartphys.2008.04.003

[21] Corda, C. (2009) Interferometric Detection of Gravitational Waves: The Definitive Test for General Relativity. International Journal of Modern Physics D, 18, 2275-2282. https://doi.org/10.1142/S0218271809015904

[22] Olmo, G.J. (2011) Palatini Approach to Modified Gravity: $f(\mathrm{R})$ Theories and Beyond. International Journal of Modern Physics D, 20, 413-462. https://doi.org/10.1142/S0218271811018925

[23] Bertolami, O., Boehmer, C.G., Harko, T. and Lobo, F.S.N. (2007) Extra Force in $f(\mathrm{R})$ Modified Theories of Gravity. Physical Review D, 75, 104016. https://doi.org/10.1103/PhysRevD.75.104016

[24] Harko, T., Lobo, F.S.N., Nojiri, S. and Odintsov, S.D. (2011) $f($ R, T) Gravity. Physical Review D, 84, 024020. https://doi.org/10.1103/PhysRevD.84.024020

[25] Brookfield, A.W., van de Bruck, C. and Hall, L.M.H. (2006) Viability of $f($ R) Theories with Additional Powers of Curvature. Physical Review D, 74, 064028.

https://doi.org/10.1103/PhysRevD.74.064028

[26] Amendola, L., Gannouji, R., Polarski, D. and Tsujikawa, S. (2007) Conditions for the Cosmological Viability of $f(\mathrm{R})$ Dark Energy Models. Physical Review D, 75, 083504. https://doi.org/10.1103/PhysRevD.75.083504

[27] Fay, S., Nesseris, S. and Perivolaropoulos, L. (2007) Can $f(\mathrm{R})$ Modified Gravity Theories Mimic a $\Lambda$ CDM Cosmology? Physical Review D, 76, 063504. https://doi.org/10.1103/PhysRevD.76.063504

[28] Amendola, L., Polarski, D. and Tsujikawa, S. (2007) Are f(R) Dark Energy Models Cosmologically Viable? Physical Review Letters, 98, 131302. https://doi.org/10.1103/PhysRevLett.98.131302

[29] Capozziello, S., Nojiri, S., Odintsov, S.D. and Troisi, A. (2006) Cosmological Viability of $f(\mathrm{R})$-Gravity as an Ideal Fluid and Its Compatibility with a Matter Dominated Phase. Physics Letters B, 639, 135-143. https://doi.org/10.1016/j.physletb.2006.06.034

[30] Santos, J., Alcaniz, J.S., Reboucas, M.J. and Carvalho, F.C. (2007) Energy Conditions in $f(\mathrm{R})$ Gravity. Physical Review D, 76, 083513. https://doi.org/10.1103/PhysRevD.76.083513

[31] Zubair, M. and Waheed, S. (2015) Energy Conditions in $f(T)$ Gravity with NonMinimal Torsion-Matter Coupling. Astrophysics and Space Science, 355, 361-369. https://doi.org/10.1007/s10509-014-2181-7

[32] Kung, J.H. (1995) $\mathrm{R}^{+} \mathrm{R}^{2}$ Gravity as $\mathrm{R}^{+}$Back Reaction. Physical Review D, 52, 6922. https://doi.org/10.1103/PhysRevD.52.6922

[33] Perez Bergliaffa, S.E. (2006) Constraining $f(\mathrm{R})$ Theories with the Energy Conditions. Physics Letters B, 642, 311-314. https://doi.org/10.1016/j.physletb.2006.10.003

[34] Hawking, S.W. and Ellis, G.F.R. (1973) The Large Scale Structure of Spacetime. Cambridge University Press, England. https://doi.org/10.1017/CBO9780511524646 
[35] Capozziello, S., Darabi, F. and Vernieri, D. (2010) Correspondence between Jordan-Einstein Frames and Palatini-Metric Formalisms. Modern Physics Letters A, 25, 3279-3289. https://doi.org/10.1142/S0217732310034456

[36] Visser, M. (2004) Jerk, Snap and the Cosmological Equation of State. Classical Quantum Gravity, 21, 2603. https://doi.org/10.1088/0264-9381/21/11/006

[37] Visser, M. (2005) Cosmography: Cosmology without the Einstein Equations. General Relativity and Gravitation, 37, 1541-1548. https://doi.org/10.1007/s10714-005-0134-8

[38] Harrison, E.R. (1976) Observational Tests in Cosmology. Nature (London), 260, 591-592. https://doi.org/10.1038/260591a0 